\title{
Intra Prediction Mode Fast Decision Algorithm Research of H.264/AVC
}

\author{
Daxing Qian ${ }^{1,}$, , Yu Fan ${ }^{2, b}$ \\ ${ }^{1}$ Dalian Neusoft University of information, Dalian 116023, China; \\ ${ }^{2}$ Chongqing University, Chongqing 400044, China. \\ ${ }^{a}$ qiandaxing@neusoft.edu.cn, ${ }^{\mathrm{b}}$ funyu405@outlook.com
}

Keywords: H.264/AVC, prediction mode decision, DCT, rate distortion model.

\begin{abstract}
Intra prediction plays an important role in H.264/AVC. The mode fast decision is a significant factor for video coding efficiency in current video coding standard. Therefore, in this paper, the authors propose a method to choose the prediction mode quickly to save coding resource. According to the frame texture characteristics, the authors choose the block size and divide one intra_16 $\times 16$ block into four intra_8x8 sub blocks with the algorithm. The sub blocks are transformed into frequency domain respectively. According to the number of zero, appropriate intra prediction mode can be decided. Furthermore, by taking advantage of direction relationship, the intra_ $4 \times 4$ sub blocks mode decision can be obtained. The experimental results demonstrate that the algorithm proposed in this paper can save coding time $30-50 \%$ while $0.04 \mathrm{~dB}$ reduction of PSNR.
\end{abstract}

\section{Introduction}

With the advance of semi-conductor and access network technologies, multimedia communications become more and more popular in our daily life. For example, we can easily enjoy the videos hosted at famous video-sharing communities (such as Youtube, Hulu, Tudou, etc) through wired or wireless networks using personal computer or mobile devices. Multimedia video carries a large amount of information, and over 60 percent information is gotten by human visual sense. How to provide the high quality of service (QoS) or coding efficiency is a crucial problem for the success of video streaming application.

H.264/AVC standard is developed by the ITU-T in collaboration with ISO. It could provide better video quality compared with previous video coding standard. Intra prediction decision calculates all of the modes and gets the best one. Although the least error can be gained, the complexity is very large. So, the fast mode decision algorithm is always the hot spot in video coding research field.

$\mathrm{Li}$ proposed a low complexity algorithm for H.264 intra prediction[1]. In his algorithm, the texture feature of an MB was determined by calculating the luma differences between each pixel and its surrounding pixels and most probable mode was selected by computing the variation of each main prediction mode, then RDO(Rate Distortion Optimized) cost was calculated among the most probable mode, its two neighboring modes and DC mode. Liu proposed a fast intra mode decision algorithm based on spatial-domain energy which was used to represent the measurement of each $4 \times 4$ sub block[2]. Variances of the sixteen $4 \times 4$ sub blocks were calculated to measure the entire variance of the MB to determine the intra encoding type.Chen proposed a new hybrid fast mode decision method[3], in which the edge based on filters are applied to some samples distributed in the block and each directional mode is related to a filtering result. Kim proposed an efficient algorithm for intra prediction using an activity map for the pixel unit of respective prediction block in conjunction with specific thresholds to determine that the MB is more suitable for Intra_4 $\times 4$ or $16 \times 16$ [4]. Thierry proposed a learning method for the distortion calculation in fast intra prediction mode selection, using partial distortion sums on a selected sub-optimal search path of sample locations in a given macroblock[5]. Kau proposed a gradient intensity-adapted algorithm with adaptive selection strategy of prediction decision[6]. In his algorithm, he applied a gradient evaluation approach to evaluate the texture orientation inside the coding block, and due to the adaptive selection strategy a subset with a variable number of prediction modes was sent to RDO process. 
In this paper, the authors propose a method to choose the prediction mode quickly to save coding resources. The experimental results demonstrate that the algorithm proposed in this paper can save a lot of time while decrease a little performance.

The rest of this paper is organized as follows. In section 2 the fast decision prediction mode algorithm is introduced. Section 3 is the coding complexity analysis. Experimental results are shown in Section 4 and finally conclusions are drawn in Section 5.

\section{Fast prediction mode algorithm}

According to the frame texture characteristics, the authors choose the intra_ $4 \times 4$ or $16 \times 16$ prediction mode. Intra_ $16 \times 16$ mode is applied to the smooth region, while intra_ $4 \times 4$ mode is used for abundant detail area. The selection of the two prediction modes depends on RDO cost function. The block whose size is $16 \times 16$ can be divided into sixteen intra_ $4 \times 4$ sub blocks. The enormous amount of computation cost on sixteen $4 \times 4$ sub-blocks will be avoided in the frame without too many details. While the frame with rich details which using intra_16 $\times 16$ block prediction algorithm will increase the RDO cost function.

To solve the above problems, the authors propose a fast decision intra mode algorithm. According to high frequency component, intra_16 $\times 16$ or $4 \times 4$ prediction mode is decided. Then the best prediction mode depends on the predicted direction relevance decision. The algorithm does not need RDO cost function to determine the coding type of macro block, and it reduces $4 \times 4$ sub block prediction range. It will reduce the intra prediction complexity.

Firstly, the authors divide a $16 \times 16$ macro block into four $8 \times 8$ sub blocks and do DCT for them. Then the coefficient matrix is quantified and the sum of zero value is counted. Lastly, we can decide the sub block is smooth or not by total number of zero. In this paper, the threshold is set to 52. If three of the four $8 \times 8$ sub blocks are smooth, the block can be considered smooth. Otherwise it will be regarded as texture complex.

Smooth macro block mode selection is simple, so this paper will not repeat them. 16x16 macro block with complex texture need to be divided into 16 intra $4 \times 4$ sub block to accurately reflect the texture.

The first five prediction modes are used with high frequency. This paper selects 0-4 mode as basic modes. The other 4 are secondary prediction mode.

We calculate the RDO function of basic modes and sort the results. If the minimum RDO is DC, it will be selected as the best mode. If the intersection angle between the smallest and second smallest $\mathrm{RDO}$ is 45 degree, the mode between them is an alternative mode. If the angle is 90 degree, the alternative mode is between the first and third smallest RDO. Compare the smallest and alternative mode, the mode with smallest RDO value is the optimal mode.

\section{Complexity analysis}

In H.264/AVC intra coding, RDO is used to calculate the optimal intra coding mode. It is calculated with the function:

$$
J=S S D+\lambda \times R
$$

$S S D$ is the sum of squared differences between the current block and its reconstruction, $\lambda$ is the Lagrange multiplier and $R$ is the bitrate.

For intra mode decision, the times of RDO cost function is calculated to measure the complexity of the algorithm. In H.264 the chroma mode is the outer loop, in order to scan all the modes of luma mode.

The calculation number is:

$$
N=\operatorname{Mode} 8 \times(\text { Mode } 4 \times 16+\text { Mode16 })
$$


Mode4 and Mode16 present the number of prediction mode of $4 \times 4$ and $16 \times 16$ luma block, respectively; Mode 8 presents the number of prediction mode of $8 \times 8$ chroma block. That means the best mode of a macro block is obtained by calculating $4 \times(16 \times 9+4)=592 \mathrm{RDO}$ cost functions.

The algorithm in this paper needs to calculate 4 RDO cost functions to intra_ $16 \times 16$ prediction mode. The luma and chroma prediction need not to calculate with RDO cost function, so just calculate 4 times. If the macro block is divided into sixteen intra_4 $\times 4$ mode, the $8 \times 8$ complex texture block needs to calculate about $4 \times 5=20$ to $4 \times 6=24$ times with RDO cost function while 5 or 6 with RDO cost function if it is smooth. The $8 \times 8$ block number of a macro block is $0,1,2$ by calculating about $4 \times 20=80,4 \times 24=96,5+3 \times 20=65$ to $6+3 \times 24=78,10+2 \times 20=50,12+2 \times$ 24=60 times respectively. The luma and chroma block are mutual independent, so the macro block needs to calculate $4 \times 50=200$ to $4 \times 96=384$ with RDO cost function. It reduces the complexity of the encoding greatly.

\section{Experimental results}

We use six test sequences to compare the performance of the proposed algorithm and the original one. The test platform is JM 8.6 and the results are shown in the following table.

Table1. H.264 fast decision intra mode performance

\begin{tabular}{ccccccc}
\hline Sequences & \multicolumn{2}{c}{ Tims(s) } & \multicolumn{2}{c}{ PSNR(dB) } & \multicolumn{2}{c}{ BR(kbits/s) } \\
\hline \multirow{4}{*}{ claire } & org & 74.524 & org & 40.99 & org & 387.98 \\
& opt & 37.142 & opt & 40.97 & opt & 415.26 \\
& Save & $50.16 \%$ & Decrease & 0.02 & Increase & $7.03 \%$ \\
mother & org & 87.070 & org & 39.06 & org & 486.38 \\
-daughter & opt & 53.450 & opt & 39.01 & opt & 521.17 \\
& Save & $38.61 \%$ & Decrease & 0.05 & Increase & $7.15 \%$ \\
& org & 90.594 & org & 39.68 & org & 567.46 \\
akiyo & opt & 52.312 & opt & 39.66 & opt & 604.02 \\
& Save & $42.26 \%$ & Decrease & 0.02 & Increase & $6.44 \%$ \\
& org & 105.225 & org & 38.18 & org & 697.91 \\
foreman & opt & 71.584 & opt & 38.15 & opt & 744.54 \\
& Save & $31.97 \%$ & Decrease & 0.03 & Increase & $6.68 \%$ \\
& org & 133.005 & org & 36.89 & org & 951.54 \\
coastguard & opt & 92.896 & opt & 38.85 & opt & 971.26 \\
& Save & $30.16 \%$ & Decrease & 0.04 & Increase & $2.07 \%$ \\
& org & 211.950 & org & 35.43 & org & 2032.58 \\
mobile & opt & 150.288 & opt & 35.35 & opt & 2057.26 \\
& Save & $29.03 \%$ & Decrease & 0.08 & Increase & $1.22 \%$ \\
\hline
\end{tabular}

The result shows that the coding time can be saved 30-50 percent, PSNR is decreased 0.02-0.08 and bitrate is increased 1-7 percent. The algorithm proposed in this paper decreases the coding complexity greatly by decreasing PSNR and increasing bitrate slightly.

\section{Summary}

In this paper, the authors propose a fast decision Algorithm to save coding resource. One $16 \times 16$ block is divided into four $8 \times 8$ sub blocks. The sub blocks are transformed into frequency domain respectively. According to the number of zero, appropriate intra prediction mode can be decided. Furthermore, by taking advantage of direction relationship, the $4 \times 4$ sub blocks mode decision can be 
obtained. The experimental results demonstrate that it can save coding time greatly while take bitrate and PSNR as small price.

\section{Acknowledgement}

This work was supported in part by the Education Department of Liaoning Province under grant L2013521.the corresponding author is Dr. Daxing Qian, Dalian Neusoft University of information, Email: qiandaxing@neusoft.edu.cn.

\section{References}

[1]. Haitao Li, Jianjun Li, G. S. Shokouh, et al. A Low Complexity Algorithm for H.264/AVC Intra Prediction. 2013 International Conference on Cyberworlds. Yokohama, Japan, 21-23 October, 2013, p. 77-81.

[2]. Yinbo Liu, Xingang Liu, Zhixin Shen, et al. Fast Intra Mode Decision Algorithm Based on Spatial-Domain Energy for H.264/AVC.2013 12th IEEE International Conference on Trust Security and Privacy in Computing and Communications (TrustCom). Melbourne, VIC, 16-18 July, 2013, p.1357-1361.

[3]. Changnian Chen, Jiazhong Chen, Tao Xia. A New Hybrid Fast Mode Decision Method for H.264/AVC Intra Coding. Proceedings of the 2013 International Conference on Machine Learning and Cybernetics. Tianjin, 14-17 July, 2013, p. 861-864.

[4]. June Kim, Youngseop Kim, Yong-Hwan Lee, et al. Efficient Compression Algorithm for H.264/AVC Intra-Prediction using an Activity Map. 2014 Eighth International Conference on Innovative Mobile and Internet Services in Ubiquitous Computing. Birmingham, United Kingdom, 2-4 July, 2014, p. 202-206.

[5]. Thierry Luhandjula, Keith L Ferguson. Sampling Point Path Selection for Fast Intra Mode Prediction. Picture Coding Symposium. Cairns, QLD, May 31-June 3 2015, p. 216-220.

[6]. Lih-Jen Kau, Jia-Wei Leng. A Gradient Intensity-Adapted Algorithm With Adaptive Selection Strategy for the Fast Decision of H.264/AVC Intra-Prediction Modes. IEEE Transactions on Circuits and Systems for Video Technology, Vol. 25, No. 6, June 2015. p. 944-957. 